\title{
¿Son rentables las empresas concentradas? El caso del sector de curtido de pieles en el Ecuador
}

\section{Are concentrated companies more profitable? The case of the tanning sector of skins in Ecuador}

Dra. Lilian Victoria Morales Carrasco es profesora/investigadora de la Universidad Técnica de Ambato (Ecuador) (lilianmora-

Ana Consuelo Córdova Pacheco es profesora/investigadora de la Universidad Técnica de Ambato (Ecuador) (anaccordova@uta. edu.ec) (http://orcid.org/0000-0001-6330-3306).

Luciano David Altamirano Espíndola es investigador de la Universidad Técnica de Ambato (Ecuador) (lucianoaltamirano435@ gmail.com) (http://orcid.org/0000-0002- 6028-1196).

Eva Cristina Lema Tituaña es investigadora de la Universidad Técnica de Ambato (Ecuador).

\section{Resumen}

El presente trabajo de investigación identifica la estructura de mercado del sector Curtido y Adobo de Pieles en el Ecuador CIIU: C151101 y tiene como objetivo establecer la relación entre rentabilidad, costos y concentración de mercado. Se calculó el índice de Herfindhal-Hirshman utilizado para casos de estructura de mercado imperfecta; y por otro lado, los índices de solvencia, liquidez, rentabilidad, para evaluar el desempeño financiero de las empresas concentradas. Además, se diseñaron cuatro modelos econométricos a los cuales se aplicaron pruebas estadísticas de idoneidad de los estimadores. Se aplicó análisis de regresión lineal simple y múltiple en varias experimentaciones a través de Mínimos cuadrados Ordinarios. De las nueve empresas del oligopolio, se identificaron cuatro empresas concentradas que controlan el $73,12 \%$ del mercado, con un margen de rentabilidad del 0,1391 promedio del oligopolio para el período de estudio 2011-2015. De los modelos econométricos desarrollados uno permitió evidenciar a través de la prueba estadística de Fisher que la concentración de mercado y los costos de producción inciden en la rentabilidad con un p-value de 0,00035, es decir, existe una relación lineal significativa de la rentabilidad del oligopolio con los costos de producción y la concentración del mercado, demostrándose así que este grupo de empresas obtienen beneficios por efecto de su capacidad para concentrar el mercado.

\begin{abstract}
The present research identifies the market structure of the Tanning and Leather Sector in Ecuador ISIC: C151101and aims to establish the relation between profitability, costs and market concentration. The Herfindhal-Hirshman index used for cases of imperfect market structure has been calculated; and on the other hand, the solvency, liquidity and profitability to evaluate the financial performance of the concentrated companies. Additionally, four econometric models have been designed to which statistical tests of adecquacy have been applied. Simple and multiple linear regression analysis has been applied in several experiments through Ordinary Least Squares. Of the nine oligopoly companies, four companies have been identified that control $73,12 \%$ of the market, with a margin of return of 0,1391 average of the oligopoly for the period of study 2011-2015. One of the developed econometric models has made possible to show through Fisher's statistical test that market concentration and production costs have an effect on profitability with a p-value of 0.00035 , meaning that there is a significant linear relation of the oligopoly with cost production and market concentration, demonstrating that this group of companies makes profits because of their ability to concentrate the market.
\end{abstract}

\section{Palabras clave I keywords}

Concentración económica, pieles, rentabilidad, crecimiento económico, mercado, beneficio. Economic concentration, skins, profitability, economic growth, market, profit.

Forma sugerida de citar: Morales Carrasco, L. V., Córdova Pacheco, A. C., Altamirano Espíndola, L. D., \& Lema Tituaña, E. C. (2018). ¿Son rentables las empresas concentradas? El caso del sector de curtido de pieles en el Ecuador. Retos Revista de Ciencias de la Administración y Economía, 15(8), 153-166.

https://doi.org/10.17163/ret.n15.2018.10 


\section{Introducción y estado de la cuestión}

En el Ecuador, existen varios sectores de actividad económica los cuales se mantienen en constante actividad empresarial, esto involucra el mismo comportamiento en los consumidores, en los productores e intermediarios y por último en el Estado. Al ser los sectores donde la oferta y la demanda emergen de manera consecutiva es evidente la presencia de tácticas competitivas que benefician tanto a compradores como a productores; lo que da como resultado la existencia de niveles de concentración altos de algunas empresas en cada sector. Este comportamiento empresarial, depende de varias aristas, que inician desde el tipo del sector, su importancia dentro de la economía nacional, el grado de involucramiento social que las empresas tengan como respuesta a su grado de responsabilidad social. Según cifras del INEC del año 2015 uno de los sectores de mayor representatividad es el Comercio al por mayor y al por menor, representado en un $46,75 \%$, le sigue el sector de Industrias Manufactureras con un participación del $16,39 \%$, Por lo expuesto anteriormente el sector económico que aporta en gran medida al empleo en el Ecuador, es el Comercio al por mayor y menor contribuyendo con el $26,15 \%$ a las fuentes de empleo a nivel nacional, seguido por el sector de manufactura que reporta el 19,50\%, es así que dentro de este sector se encuentra la actividad empresarial de Curtido y Adobo de cueros en el Ecuador (Lema, 2017).

Este sector se desarrolla hace 70 años en el Ecuador, de manera artesanal y al devenir de los años empezó a industrializarse, como resultado de la proyección urbanística que se acrecentó en las últimas décadas; a partir del año 2013 y 2014 las ventas del sector curtido y adobo de cuero tuvieron un crecimiento significativo en relación al 2011, donde se registra un crecimiento del 24,82\% y 17,86\%, para luego en los años subsiguientes 2015 y 2016 denotar un decrecimiento del sector (Lema, 2017). Es necesario diferenciar que en el Sector de Fabricación de cuero en el Ecuador; hay dos subsectores de actividad económica; el primero de Curtido y adobo de cueros, fabricación de maletas, bolsos de mano y artículos de talabartería y guarnicionería y el último de Fabricación de calzado, siendo este el más representativo en ventas a nivel nacional aportando con el 86,74\% al sector según cifras del año 2015, dicho lo anterior el primer subsector representa el 10,09\%; en realidad si bien es cierto el sector de curtido y adobo de cuero no es muy representativo en la productividad ni en la aportación al empleo sin embargo es necesario conocer el grado de afectación al consumidor en el caso de que el sector este concentrado (Lema, 2017).

En este contexto el objetivo de este trabajo es demostrar si los niveles de concentración tiene un efecto de respuesta en los niveles de rentabilidad, de las empresas, si esto es así, las empresas dominantes, mantendrían un estado de conformidad lo cual a largo plazo limita notablemente la innovación como consecuencia de la incipiente interacción empresarial, fomentado la riqueza empresarial y generando un retroceso en materia de equidad y crecimiento económico del sector, identificado a través de los indicadores financieros aplicados a las empresas. De igual manera en la investigación de Cardona Olaya, Martínez Carvajal, Velásquez Restrepo, \& López Fernández (2015) se analiza el desempeño financiero de las empresas colombianas del sector de marroquinería y cuero, el presente estudio lleva consigo una aplicación descriptiva de la información documental, donde se aborda catorce indicadores de rentabilidad, apalancamiento, administración de activos, generación de valor, y liquidez por actividad 
económica y por año. Con respecto al indicador de prueba ácida este considera las cuentas del activo corriente excepto el inventario para dividirlo con el pasivo corriente, es una medida mas eficaz cuando el inventario no tiene una capacidad inmediata de convertirse en efectivo, de este estudio se detectaron diferencias en el desempeño financiero de las cuatro actividades económicas que conforman el sector. La investigación de Castaño Ríos et al. (2016) aplican indicadores financieros de rentabilidad (ROE-ROI) de las empresas de cemento, cal y yeso del departamento de Antioquia y su contribución con el PIB manufacturero durante los periodos 2008-2013, utilizando un análisis cuantitativo-descriptivo, llega a determinar una relación directa entre las tres variables (ROE-ROI y PIB) las cuales le permite indagar sobre si las empresas más rentables aportan al PIB. La utilización de variables financieras en la aplicación de estudios de correlación es amplia y permite evaluar financieramente a los sectores en varias actividades económicas donde surgen empresas grandes, pequeñas y medianas donde el poder de dirección es un factor de éxito en las empresas, el efecto inmediato se ve representado en las ventas, que conduce a empresas con un alto poder de mercado, sujeto a calificarlos como mercados concentrados.

La presencia de mercados concentrados es un acontecimiento presente en todo el mundo, lo cual significa una constante lucha entre los entes de control y las empresas cuyas condiciones en el mercado prometen ser las más favorables para los grandes grupos empresariales y las más nocivas para los ciudadanos en general. Es así que las condiciones de concentración difieren acorde a la realidad y a las legislaciones de cada país, sin embargo, se busca el mismo objetivo, tratar de proteger a los sectores más vulnerables de la población a padecer los efectos negativos del ejercicio del poder de mercado que en algunos casos son nocivas y desleales, los estudios que se detallarán a continuación estructuran un análisis inferencial y descriptivo abordando sectores concentrados de la economía a nivel mundial y regional, a los cuales se aplica una metodología econométrica que permite identificar los efectos y causas que conllevan a concentrar el mercado.

A un mercado que presente una cantidad limitada de empresas activas se le conoce como de competencia imperfecta, el cual se presenta como monopolio, oligopolio o monopsonio. El oligopolio es una de las formas de mercado de competencia imperfecta más comunes y Jaén et al. (2013) lo caracterizan como una situación intermedia entre competencia perfecta y monopolio en la cual no necesariamente se ejerce poder de mercado. Esto debido a que la demanda de una empresa se relaciona con la de la competencia; es decir que no es tan independiente a su entorno como la demanda de un monopolio. De esta forma, en un entorno de competencia perfecta las empresas se ven en la posibilidad de aplicar estrategias de control, aspecto que nace propiamente de la estructura de su mercado.

Las compañías aplican estrategias para adquirir dominio sobre el sector en el que se desenvuelven, mismas que pueden basarse en el precio o no. Dentro de las estrategias basadas en el precio, según afirma Jaén (2013), se encuentra la discriminación de precios en primer, segundo y tercer grado, mientras que dentro de las estrategias no basadas en el precio se pueden clasificar a las ventas por lotes, conjuntas o de cesta de bienes y las ventas ligadas. El privilegio de contar con pocos competidores en el mercado posibilita la adopción de ciertas estrategias de dominio; sin embargo este privilegio 
se ve limitado por diversos factores. Un caso primordial es el de la presencia de barreras de entrada, cuyo entendimiento es inherente al ejercicio de poder de mercado y la concepción de oligopolio o monopolio sería vacía sin tomar en consideración este factor.

La implantación de barreras de entrada por parte de las titulares, puede fundamentarse en el amplio conocimiento que las empresas tengan de los proveedores y en ocasiones, de ciertas ventajas que estas empresas les puedan ofrecer. No necesariamente aquello puede significar una acción ilegal, sin embargo, es una estrategia de implantación de barreras al ingreso de posibles competidores.

Por otro lado, la estructura de mercado oligopólico determina que hay varios factores que los competidores manejan como estrategia de penetración en el mercado que influyen en el consumidor de manera directa y que formalizan su cuota de mercado. Históricamente la estructura del mercado en el sector ha sido concentrada, siendo las empresas multinacionales las que siempre han controlado el mercado y al ser la producción y comercialización bananera uno de los sectores económicos más importantes en la costa caribeña colombiana, es de gran importancia describir el comportamiento de las empresas y de los consumidores que controlan el mercado, para aquello utiliza el índice Herfindahl-Hirschman, conjuntamente con un modelo del comportamiento de las estrategias que realizan las comercializadoras siguiendo la teoría de juegos y un modelo de regresión lineal múltiple del precio al productor en función del nivel de concentración del sector y del comportamiento cíclico de los precios del banano. Seguido a ello, se realiza un seguimiento a los efectos sociales de la concentración de mercado, de manera que se pueda determinar si la estructura de mercado existente genera efectos positivos o negativos en la población. demostró que el mercado de comercialización de banano se encuentra concentrado y que existe una relación inversa entre los precios al productor y la concentración de mercado expresada a través del Índice de concentración de Herfindahl-Hirschman. Las pérdidas sociales generadas por la concentración y el poder de mercado ejercido por las comercializadoras de banano son considerables.

Continuando con la posibilidad de ejercer poder de mercado a través de la formación de precios, en el contexto de la producción mundial de aceite de palma et al. (2013) buscan estudiar la estructura de mercado de la producción mundial de aceite de palma y se plantea el análisis de la influencia de esta variable sobre el nivel de precios del producto, se realiza un estudio de características causales por medio de un análisis de correlación entre los niveles de concentración de mercado del sector y los precios del aceite de palma establecidos en el mercado del aceite de palma. Con el objeto de efectuar dicho estudio, se realiza un análisis estadístico de correlación conjuntamente con la descripción del Índice de Herfindahl-Hirschman (IHH), el índice de Dominación (ID), la tasa de concentración (CR) y del precio del aceite de palma en el mercado internacional. Se encontró una considerable relación existente entre los precios internacionales del producto y el grado de concentración de mercado, sin embargo, no se observó la presencia de abuso de poder de mercado, lo cual asume que una estructura concentrada no necesariamente implica ejercer poder sobre este como un monopolio u oligopolio. Esto se debe a la facilidad que existe de incursionar en el sector, con lo cual se observó un cambio del liderazgo del mercado, puesto que pasó de estar dominado por un solo productor a encontrarse liderado por dos productores. 


\section{Material y métodos}

Para la realización del presente trabajo de investigación, en primer lugar, se procedió a identificar el sector y a las empresas que desarrollan actividades de curtiduría. Esta actividad se encuentra identificada por el Servicio de Rentas Internas (SRI) bajo el código CIIU: C142001 acorde a la Clasificación Nacional de Actividades Económicas según el Instituto Nacional de Estadística y Censos (2012), sin embargo, conforme a la base de datos de la Superintendencia de Compañías del Ecuador las empresas que realizan actividades de curtiduría se encuentran identificadas bajo el código CIIU: C151101, debido a esto, se identificaron a las empresas según el criterio de la Superintendencia de Compañías y para su contraste con la totalidad de ventas del sector, se tomó en cuenta el criterio del SRI y se homologó en base a la descripción de ambas. Las empresas que desarrollan actividades y se encuentran activas dentro del sector al año 2017, son Curtiduría Tungurahua S.A., Curtiembre Renaciente S.A., Servicueros S.A., Tenería San José Cía. Ltda., Tenería Díaz Cía Ltda., Cabaro Cía. Ltda., Curtigual S.A., Promepell S.A. y Proinpiel S.A.

Para el análisis de las ventas totales del sector, el estudio se basó en las estadísticas otorgadas por el SRI derivadas del formulario de declaración al impuesto al Valor Agregado (IVA) de sociedades y personas naturales, puesto que brinda información referente a las ventas gravadas con el IVA, que representan la totalidad de transacciones comerciales que las empresas realizan en el Ecuador.

Para el análisis descriptivo se utilizaron los datos de Ingresos, Ventas, Costos de producción, Activos fijos y Pasivo a largo plazo durante el período 2011-2015 de las cuatro empresas de mayor volumen de ventas del sector (Curtiduría Tungurahua, Servicueros, S.A., Curtiembre Renaciente S.A. y Tenería San José Cía. Ltda.). Además, se presentan indicadores de solvencia, liquidez y rentabilidad, para lo cual se adoptó a la cuenta endeudamiento patrimonial como un indicador de Solvencia, a la liquidez corriente como un indicador de Liquidez y al margen bruto de rentabilidad como índice de Rentabilidad. La utilización de las razones financieras que incluye los métodos de cálculo e interpretación, permiten al gestor financiero, el análisis del rendimiento, solvencia y liquidez de las empresas en el continuo ejercicio de la actividad de manufactura en cualquier sector. Por otro lado hay que considerar que la correcta gestión de la administración del efectivo permite determinar si las empresas analizadas cuentan con suficientes recursos disponibles a corto plazo para cumplir con las obligaciones. Los acreedores a corto plazo de la empresa, consideran que mientras más alta sea la razón del circulante es mucho mejor para los acreedores en calidad de proveedores ya que puede mostrar una alta liquidez. De igual manera las razones de solvencia involucran la medición de la capacidad de las empresas, para satisfacer sus obligaciones a largo plazo. Para la elaboración de estos indicadores financieros, fue necesario identificar a las cuentas del Activo y Pasivo corriente, los Activos y Pasivos Totales y la cuenta del patrimonio, de manera que se puedan efectuar las relaciones antes mencionadas; en la Tabla 1 se presenta un resumen de los indicadores a analizarse y su cálculo correspondiente. 
Tabla 1. Indicadores

\begin{tabular}{|l|c|}
\hline \multicolumn{1}{|c|}{ Índice } & Fórmula \\
\hline Endeudamiento patrimonial & $=\frac{\text { Pasivo no corriente }}{\text { Patrimonio }}(1)$ \\
\hline Liquidez corriente & $=\frac{\text { Activo corriente }}{\text { Pasivo corriente }}(2)$ \\
\hline Margen bruto & $=\frac{\text { Ventas }- \text { Costo de ventas }}{\text { Ventas }}(3)$ \\
\hline Índice de Hirschman-Herfindahl & $=\sum(\text { Cuota de mercado })^{2} \times 10.000$ \\
\hline
\end{tabular}

Fuente: Tanaka (2005); Caballero (2014), Aching (2006), Parkin \& Loría (2010).

La principal hipótesis de investigación consiste en que la concentración de mercado se relaciona con el desempeño de las empresas del sector de fabricación de pieles curtidas en el Ecuador. En consecuencia, se pretende identificar el grado de concentración de mercado que presenta el sector y contrastar el hecho de que el índice de concentración de Herfindahl-Hirschman incide estadísticamente en la rentabilidad de las compañías y viceversa. Esto permitirá conocer si existe concentración en el mercado y si las empresas ejercen poder sobre el mismo de manera que se de explicación al comportamiento adoptado por las empresas dedicadas en el contexto competitivo.

En lo que respecta al análisis econométrico, se estructuraron dos modelos de regresión lineal simple y tres modelos de regresión lineal múltiple, de los cuales se identificarán a cuatro variables de interacción: la rentabilidad que se encuentra expresada a través del margen bruto de rentabilidad, la concentración de mercado representada por el índice de concentración de Herfindahl-Hirschman, el crecimiento económico como tasa de variación del PIB y los costos de producción de las cuatro compañías más representativas del sector.

El primer modelo se encontró estructurado por la rentabilidad (Margen bruto) en función de la concentración de mercado (Índice de Herfindahl-Hirschman) como lo establece Duarte (2014) al presentar un modelo de regresión lineal simple representando a la rentabilidad o utilidad bruta como variable dependiente y a los precios por unidad producida como variable independiente. En este caso, a diferencia de lo establecido por Duarte (2014) en el primer modelo se toma como variable independiente a la concentración de mercado, con el objetivo de determinar la incidencia y el grado de explicación de la rentabilidad de las cuatro empresas más representativas del sector en función del grado de concentración del sector, que básicamente busca el mismo objetivo que Duarte (2014).

$$
Y=\beta_{0}+\beta_{1} X_{1}+u
$$

Donde: 
$\mathrm{Y}=$ Rentabilidad (Margen bruto).

$\mathrm{X}_{1}=$ Concentración de mercado (Índice de Herfindahl - Hirschman).

$\mathrm{u}=$ Error de perturbación.

$\beta_{0}$ y $\beta_{1}=$ Estimadores.

El segundo modelo explica a la concentración de mercado, es decir, al índice de Herfindahl-Hirschman en función de la Rentabilidad o margen bruto, dicho de otra forma, el modelo número 2 es la versión inversa del modelo de regresión lineal anterior, esto con el objetivo de analizar el grado de explicación de la concentración de mercado en función de la rentabilidad que puedan obtener las empresas líderes del sector.

$$
X_{1}=\beta_{0}+\beta_{1} Y+u
$$

Donde:

$\mathrm{X}_{1}=$ Concentración de mercado (Índice de Herfindahl - Hirschman).

$\mathrm{Y}=$ Rentabilidad (Margen bruto).

$\mathrm{u}=$ Error de perturbación.

$\beta_{0}$ y $\beta_{1}=$ Estimadores.

El modelo número 3 se diseñó por las tasas de variación del margen bruto de rentabilidad en función de la tasa de variación del Índice de concentración de Herfidahl-Hirschman y del crecimiento económico expresado a través de la tasa de crecimiento del PIB del Ecuador. Este modelo de regresión lineal múltiple pretende analizar la incidencia y el grado de explicación de los márgenes de rentabilidad de las principales empresas del sector de curtiduría y adobo de pieles en el Ecuador, en función de la concentración del mercado y del comportamiento de la economía en su contexto. Cabe recalcar que nuevamente se adopta el criterio de Duarte (2014) de establecer a la rentabilidad como variable dependiente, salvo que para este modelo se incorporó la interacción de la tasa de crecimiento del PIB.

$$
\mathrm{Y}=\beta_{0}+\beta_{1} \mathrm{X}_{1}+\beta_{2} \mathrm{X}_{2}+\mathrm{u}
$$

Donde:

$\mathrm{Y}=$ Tasa de variación de la rentabilidad (Margen bruto).

$\mathrm{X}_{1}=$ Tasa de variación del índice de Herfindahl - Hirschman.

$\mathrm{X}_{2}=$ Tasa de crecimiento del PIB.

$\mathrm{u}=$ Error de perturbación.

$\beta_{0} \mathrm{y} \beta_{1}=$ Estimadores. 
El modelo número 4 se estructuró con el margen bruto de rentabilidad como variable dependiente y al índice de Herfindahl-Hirschman, la tasa de variación del PIB y los costos de producción como variables independientes. En este modelo, se incorporó a la variable de costos de producción adoptando el criterio de Duarte (2014) que no necesariamente tratan a esta variable como parte de un modelo econométrico pero la toman en cuenta para su análisis descriptivo. Se optó por integrar a esta variable dentro del modelo, con el objeto de identificar de una mejor manera los efectos de la concentración y del crecimiento económico sobre los niveles de rentabilidad dentro del sector estudiado ya que el margen bruto de rentabilidad depende directamente de los costos de producción.

$$
Y=\beta_{0}+\beta_{1} X_{1}+\beta_{2} X_{2}+\beta X_{3}+u
$$

Donde:

$\mathrm{Y}=$ Rentabilidad (Margen bruto).

$\mathrm{X}_{1}=$ Concentración de mercado (Índice de Herfindahl - Hirschman).

$\mathrm{X}_{2}=$ Tasa de crecimiento del PIB.

$\mathrm{X}_{3}=$ Costos de producción.

$\mathrm{u}=$ Error de perturbación.

$\beta_{0}, \beta_{1}, \beta_{2} y \beta_{3}=$ Estimadores.

El modelo número 5 resulta directamente del modelo número 4, puesto que se aplicó el test de colinealidad a través del Factor de Inflación de Varianza (VIF) por sus siglas en inglés, lo que identificó que la variable tasa de crecimiento del PIB presentó colinealidad, por lo cual se procedió a desestimar a esta variable para el desarrollo del modelo econométrico.

$$
Y=\beta_{0}+\beta_{1} X_{1}+\beta_{3} X_{3}+u
$$

Donde:

$\mathrm{Y}=$ Rentabilidad (Margen bruto).

$\mathrm{X}_{1}=$ Concentración de mercado (Índice de Herfindahl - Hirschman).

$\mathrm{X}_{3}=$ Costos de producción.

$\mathrm{u}=$ Error de perturbación.

$\beta_{0}, \beta_{1}$ y $\beta_{3}=$ Estimadores.

A cada uno de los modelos se aplicaron pruebas estadísticas que permitan determinar la idoneidad de los estimadores para la realización de predicciones y de contraste de hipótesis a través de los modelos propuestos como: el test de no linealidad, el test de especificación de RESET de Ramsey, de Heterocedasticidad de White, la prueba de Autocorrelación de Durbin Watson y de Breusch-Godfrey, el test de Colinealidad del 
Factor de Inflación de Varianza (VIF) y de normalidad de los residuos de Jarque-Bera. Debido al tamaño de la muestra y de la cantidad de regresoras no fue posible efectuar ciertos tests estadísticos a algunos de los modelos propuestos, como es el caso del test de Autocorrelación de Durbin Watson y de Breusch-Godfrey, el test de RESET de Ramsey y la prueba de Heterocedasticidad de White.

\section{Análisis y resultados}

En la presente investigación se busca realizar un análisis de la estructura del mercado del sector curtido y adobo de cuero CIIU: 111501 en el Ecuador. Se describen las ventas de cada una de las compañías, la cuota de mercado y su índice de concentración respectivo, con lo cual se puede observar el comportamiento de las empresas que lideran el sector.

Tabla 2. Ventas de las cuatro empresas concentradas del sector curtido y adobo de pieles

\begin{tabular}{|l|r|r|r|r|r|r|}
\hline \multicolumn{7}{|c|}{ Ventas } \\
\hline Empresas & $\mathbf{2 0 1 1}$ & $\mathbf{2 0 1 2}$ & \multicolumn{1}{c|}{$\mathbf{2 0 1 3}$} & \multicolumn{1}{c|}{$\mathbf{2 0 1 4}$} & $\mathbf{2 0 1 5}$ & \multicolumn{1}{c|}{ Promedio } \\
\hline A & 7157900,52 & 9400968,82 & 12468131,30 & 13827271,30 & 12433762,50 & 11057606,89 \\
\hline B & 2926582,98 & 3159810,39 & 3681256,50 & 5271600,06 & 5555130,41 & 4118876,07 \\
\hline C & 1898848,00 & 3116853,76 & 4205653,05 & 3245023,03 & 2658223,37 & 3024920,24 \\
\hline D & 2870574,70 & 2826163,04 & 4205653,05 & 3948487,60 & 2653702,29 & 3300916,14 \\
\hline Total & 14853906,20 & 18503796,01 & 24560693,90 & 26292381,99 & 23300818,57 & 21502319,33 \\
\hline
\end{tabular}

Fuente: Elaboración propia a partir de los datos suministrados por la Superintendencia de Compañías y SRI.

Las ventas por empresa del oligopolio en promedio representan el 73,42\% de las ventas totales realizadas por todo el sector desde el año 2011 hasta el 2015, ubicándose a la empresa A, como la compañía con mayores ventas en el mercado alcanzando un promedio en ventas de 11057 606,89 durante el período analizado. En segundo lugar, se encuentra la empresa B con unas ventas promedio de 4118 876,07, mientras que en tercero y cuarto lugar se encuentran D y C con unas ventas promedio de 3300 916,14 y 3024920,24 respectivamente.

Tabla 3. Ventas, Cuota de Mercado e Índice de concentración del sector

\begin{tabular}{|l|r|r|r|}
\hline Empresas & Ventas 2015 & $\begin{array}{c}\text { Cuota de mercado } \\
\mathbf{2 0 1 5}\end{array}$ & $\begin{array}{c}\text { Índice de } \\
\text { Hirschman- } \\
\text { Herfindahl 2015 }\end{array}$ \\
\hline A & 12433762,50 & $39,02 \%$ & 1522,27 \\
\hline B & 5555130,41 & $17,43 \%$ & 303,86 \\
\hline C & 2658223,37 & $8,34 \%$ & 69,58 \\
\hline D & 2653702,29 & $8,33 \%$ & 69,34 \\
\hline F & 1718142,73 & $5,39 \%$ & 29,07 \\
\hline
\end{tabular}




\begin{tabular}{|l|r|r|r|}
\hline G & 366862,50 & $1,15 \%$ & 1,33 \\
\hline H & 280767,04 & $0,88 \%$ & 0,78 \\
\hline I & 124085,87 & $0,39 \%$ & 0,15 \\
\hline Otras empresas & 5202307,02 & $16,32 \%$ & 266,49 \\
\hline
\end{tabular}

Fuente: Elaboración propia a partir de los datos suministrados por la Superintendencia de Compañías del Ecuador

Como se aprecia en la Tabla 3, la empresa que lidera el mercado representa el $39,02 \%$ de las ventas del sector de curtido y adobo de cuero. El segundo lugar, representando por el $17,43 \%$ de las ventas totales en el mercado, seguidamente $8,34 \%$ y $8,33 \%$ en el tercer y cuarto lugar de cuota de mercado respectivamente. En el sector existen 11 empresas activas registradas como sociedades ( 2 de las cuales no registran ventas en sus estados financieros durante el año 2015), mientras que las empresas establecidas como personas naturales representan el $16,32 \%$.

Tabla 4. Solvencia por empresa

\begin{tabular}{|l|c|r|r|r|r|c|}
\hline \multicolumn{7}{|c|}{ Solvencia-Endeudamiento patrimonial } \\
\hline Empresas & $\mathbf{2 0 1 1}$ & $\mathbf{2 0 1 2}$ & $\mathbf{2 0 1 3}$ & $\mathbf{2 0 1 4}$ & $\mathbf{2 0 1 5}$ & Promedio \\
\hline A & 2,76 & 2,33 & 2,47 & 2,52 & 1,57 & 2,33 \\
\hline B & 1,63 & 1,61 & 0,85 & 1,09 & 1,65 & 1,37 \\
\hline C & 1,11 & 2,1 & 2,38 & 2,49 & 3,25 & 2,27 \\
\hline D & 3,64 & 1,74 & 2,38 & 1,48 & 1,6 & 2,17 \\
\hline
\end{tabular}

Fuente: Elaboración propia a partir de los datos suministrados por la Superintendencia de Compañías del Ecuador

La empresa más solvente del oligopolio es sin duda la empresa B que en promedio del período analizado registró un endeudamiento patrimonial de 1,37 que, aunque no es un valor óptimo de solvencia es el más bajo observado en todo el oligopolio. La segunda empresa más solvente es la D que, aunque presentó alto índice de endeudamiento patrimonial durante el año 2011, experimentó un decrecimiento marcado durante años posteriores. Por otro lado, las empresas A y C presentan los más altos índices de insolvencia promedio del período, lo cual muestra un alto financiamiento utilizado potencialmente para expandirse en el mercado, ya que estas dos empresas son las que mayor capacidad instalada poseen de todo el oligopolio.

Tabla 5. Liquidez corriente por empresa

\begin{tabular}{|l|c|c|c|c|c|c|}
\hline \multicolumn{7}{|c|}{ Liquidez-Liquidez corriente } \\
\hline Empresas & $\mathbf{2 0 1 1}$ & $\mathbf{2 0 1 2}$ & $\mathbf{2 0 1 3}$ & $\mathbf{2 0 1 4}$ & $\mathbf{2 0 1 5}$ & Promedio \\
\hline A & 1,52 & 1,91 & 1,2 & 1,22 & 1,67 & 1,504 \\
\hline B & 2,07 & 2,35 & 1,9 & 1,91 & 2,58 & 2,162 \\
\hline C & 1,93 & 1,18 & 1,63 & 1,17 & 1,18 & 1,418 \\
\hline D & 1,25 & 0,99 & 1,63 & 1,33 & 2,14 & 1,468 \\
\hline
\end{tabular}

Fuente: Elaboración propia a partir de los datos suministrados por la Superintendencia de Compañías del Ecuador 
La empresa con mayor liquidez en el sector es la empresa B, puesto que en promedio registró un valor de liquidez corriente de 2,16 en el período analizado, el cual es superior al límite óptimo. Durante los años 2013 y 2014 la empresa B presentó una reducción de sus márgenes de liquidez, lo cual es atribuible a la estrategia de expansión. Finalmente, se encuentran las empresas C y D las cuales presentaron en promedio durante todo el período analizado los índices más bajos de liquidez corriente, siendo estos de 1,42 y 1,47 respectivamente, mientras que la empresa A se mantuvo en los límites óptimos de liquidez.

Tabla 6. Rentabilidad, margen bruto por empresa

\begin{tabular}{|c|c|c|c|c|c|c|}
\hline \multicolumn{7}{|c|}{ Rentabilidad-Margen bruto } \\
\hline Empresas & 2011 & 2012 & 2013 & 2014 & 2015 & Promedio \\
\hline A & 0,09 & 0,16 & 0,17 & 0,12 & 0,14 & 0,14 \\
\hline B & 0,17 & 0,18 & 0,16 & 0,13 & 0,1 & 0,15 \\
\hline $\mathrm{C}$ & 0,31 & 0,31 & 0,28 & 0,33 & 0,27 & 0,30 \\
\hline $\mathrm{D}$ & 0,17 & 0,13 & 0,27 & 0,15 & 0,06 & 0,16 \\
\hline
\end{tabular}

Fuente: Elaboración propia a partir de los datos suministrados por la Superintendencia de Compañías

La empresa más rentable del oligopolio es la empresa $\mathrm{C}$, registrando un valor del margen bruto de rentabilidad promedio del período de 0,30. Los márgenes de rentabilidad de la empresa mantuvieron un comportamiento estable sin claras tendencias ascendentes o decrecientes, salvo el año 2014, en el cual se registró el valor más alto nivel de rentabilidad de la compañía, siendo este de 0,33. Por otro lado, la empresa con menor margen bruto de rentabilidad es la empresa A, registrando un promedio del margen bruto de 0,14 durante todo el período analizado. La empresa mantuvo una tendencia decreciente de su rentabilidad, en especial a partir del año 2013, con lo cual se registró un importante descenso de su rentabilidad para el año 2014 y una leve recuperación para el 2015.

Tabla 7. Ventas, Costos de producción, rentabilidad y concentración de mercado de las empresas concentradas del sector

\begin{tabular}{|l|c|c|c|c|}
\hline Años & $\begin{array}{c}\text { Ventas } \\
\text { de las empresas } \\
\text { concentradas }\end{array}$ & $\begin{array}{c}\text { Costos } \\
\text { de producción } \\
\text { de las empresas } \\
\text { concentrada }\end{array}$ & $\begin{array}{c}\text { Rentabilidad } \\
\text { (Margen bruto) } \\
\text { de las empresas } \\
\text { concentrada }\end{array}$ & $\begin{array}{c}\text { Concentración } \\
\text { de mercado }\end{array}$ \\
\hline 2011 & 14853906,20 & 13246396,87 & 0,1082 & 1423,10 \\
\hline 2012 & 12560779,21 & 10451712,84 & 0,1679 & 1893,34 \\
\hline 2013 & 16149387,80 & 13461679,91 & 0,1664 & 2186,64 \\
\hline 2014 & 19098871,36 & 16725817,56 & 0,1243 & 2061,77 \\
\hline 2015 & 17988892,91 & 15673051,95 & 0,1287 & 2010,68 \\
\hline
\end{tabular}

Fuente: Superintendencia de Compañías 
Tabla 8. Tasas de variación de ventas, costos de producción, rentabilidad y concentración de mercado del sector

\begin{tabular}{|l|c|c|c|c|}
\hline Indicadores & $\begin{array}{c}\text { Tasa de variación } \\
\text { Ventas de las } \\
\text { principales } \\
\text { empresas }\end{array}$ & $\begin{array}{c}\text { Tasa de variación } \\
\text { Costos de } \\
\text { producción de } \\
\text { las principales } \\
\text { empresas }\end{array}$ & $\begin{array}{c}\text { Tasa de variación } \\
\text { Rentabilidad de las } \\
\text { principales empresas } \\
\text { (Margen bruto) }\end{array}$ & $\begin{array}{c}\text { Tasa } \\
\text { de variación } \\
\text { Concentración } \\
\text { de mercado }\end{array}$ \\
\hline 2012 & $-15,44 \%$ & $-21,10 \%$ & $55,15 \%$ & $33,04 \%$ \\
\hline 2013 & $28,57 \%$ & $28,80 \%$ & $-0,88 \%$ & $15,49 \%$ \\
\hline 2014 & $18,26 \%$ & $24,25 \%$ & $-25,34 \%$ & $-5,71 \%$ \\
\hline 2015 & $-5,81 \%$ & $-6,29 \%$ & $3,61 \%$ & $-2,48 \%$ \\
\hline
\end{tabular}

Fuente: Elaboración propia a partir de los datos suministrados por la Superintendencia de Compañías

Los costos de producción del oligopolio presentados en la Tabla $\mathrm{N}^{\circ} 5$ muestran un comportamiento armónico con las ventas que no es el resultado de una variación en los precios. Para el año 2014 se evidenció una importante reducción del margen bruto de rentabilidad y una leve recuperación experimentada durante el año 2015, lo cual podría ser atribuible a la desaceleración de la economía ecuatoriana en los tres años anteriores.

La concentración de mercado expresada a través del índice de HerfindahlHirschman experimentó un marcado crecimiento durante los años 2011, 2012 y 2013, es decir que, el oligopolio estudiado mantuvo un proceso expansivo de captura del mercado resultando en un incremento de la cuota de participación en el sector, para que posteriormente durante los años 2014 y 2015 la concentración del oligopolio decreciera. Durante el transcurso del año 2011 al año 2013 el índice de concentración de HerfindahlHirschman creció en promedio un 18,83\%, mientras que desde el año 2013 hasta el 2015 el indicador decreció en promedio un 4,11\%, con lo que es apreciable la expansión del oligopolio con respecto al año 2011.

A continuación, se presenta el modelo econométrico que explica la rentabilidad del oligopolio con las variables concentración y costos de producción. El modelo que se escoge es el resultado de 4 experimentaciones, en función de los modelos que constan en el apartado de metodología. Se desestimó a la variable tasa de crecimiento del PIB debido a que presentó colinealidad registrando un valor superior a 10 a través del test del Factor de Inflación de Varianza (VIF), siendo este de 14,943.

Tabla 9. Rentabilidad en función de la concentración de mercado y de los costos de producción

\begin{tabular}{|l|l|l|l|l|}
\hline \multicolumn{1}{|c|}{ Variable } & Coeficiente & $\begin{array}{c}\text { Error } \\
\text { estándar }\end{array}$ & Estadístico t & $\begin{array}{c}\text { Valor de } \\
\text { probabilidad }\end{array}$ \\
\hline Constante & $1,1964 \mathrm{E}-01$ & $8,6279 \mathrm{E}-03$ & 13,87 & 0,0052 \\
\hline Concentración de mercado (IHH) & $7,7137 \mathrm{E}-05$ & $4,0101 \mathrm{E}-06$ & 19,24 & 0,0027 \\
\hline Costos de producción & $-9,22 \mathrm{E}-09$ & $4,85808 \mathrm{E}-10$ & $-18,98$ & 0,0028 \\
\hline $\begin{array}{l}\text { Coeficiente de determinación } \\
\text { corregido: }\end{array}$ & 0,9929 & & & \\
\hline Estadístico de Fisher: & 280,65 & Valor p: & 0,0036 & \\
\hline
\end{tabular}




\begin{tabular}{|l|l|l|l|l|}
\hline Test de no linealidad & & & & \\
\hline $\begin{array}{l}\text { Hipótesis nula: La relación es } \\
\text { lineal }\end{array}$ & Valor p: & 0,0821 & & \\
\hline $\begin{array}{l}\text { Test de normalidad de los resi- } \\
\text { duos de Jarque Bera }\end{array}$ & & & & \\
\hline $\begin{array}{l}\text { Hipótesis nula: el error se } \\
\text { distribuye normalmente }\end{array}$ & Valor p: & 0,7832 & & \\
\hline
\end{tabular}

Fuente: Elaboración propia a partir de los Datos estadísticos descritos en la Tabla 7

Como se puede observar en la Tabla 7, la concentración de mercado incide en la rentabilidad del oligopolio, puesto que se evidenció un valor p estadísticamente significativo con un valor de 0,0027. De igual manera, se determinó que los costos de producción también inciden en la rentabilidad del oligopolio, puesto que observó un valor p del estimador estadísticamente significativo, de 0,0028. Además, se registró un coeficiente de determinación de 0,9929, lo que implica que, la concentración de mercado y los costos de producción explican en un 99,29\% a la rentabilidad del oligopolio.

A través del test de no linealidad se determinó que las variables del modelo se relacionan linealmente, puesto que se observó un valor p del estadístico no significativo con un valor de 0,081 . De igual manera, se evidenció que los residuos del modelo se distribuyen normalmente, puesto que se observó un valor del estadístico de Jarque-Bera de 0,7832, con lo que se determina que los estimadores son confiables, sesgados y eficientes.

\section{Discusión y conclusiones}

La presencia de concentración de mercado generalmente se ve relacionada con la potestad de implantar precios por encima de lo justo a costa del bienestar del consumidor; sin embargo esto no siempre podría ocurrir. Un caso evidente de presencia de concentración y ejercicio de poder de mercado lo evidencia Duarte (2014), al registrar una relación entre la rentabilidad y el nivel de concentración. En contraste, Navarro, Ocampo \& Saumeth (2013) también registraron incidencia de la concentración de mercado sobre la rentabilidad dentro del sector de fabricación de aceite de Palma; sin embargo, la investigación descartó cualquier posibilidad de ejercicio de poder de mercado debido a la inexistencia de barreras de entrada. El presente estudio muestra hallazgos similares a lo observado por Duarte (2014) al evidenciarse una relación entre la rentabilidad y el índice de concentración de Herfindahl-Hirschman y se considera la presencia de ejercer poder de mercado.

El desempeño empresarial esta ligado a la ciclicidad de la economía en su conjunto; sin embargo, los sectores de actividad económica que mantienen barreras de entrada son reacios a afectarse por los procesos recesivos. La investigación observó la inexistencia de relación entre la tasa de crecimiento del PIB y la rentabilidad de las curtiembres. Dicha independencia entre variables se relaciona con la existencia de barreras de entrada, debido a que un sector de difícil acceso tiende a resistir de mejor manera el decrecimiento de la actividad productiva. Castaño et al. (2016) por su parte, evidenciaron una fuerte relación entre la rentabilidad de las empresas cementeras y el PIB del departamento de Antioquia; es decir que, se evidenció un comportamiento cíclico del sector. 
Por lo general el mercado de fabricación de cemento posee importantes competidores en el mercado internacional, lo que supone la presencia de barreras de entrada débiles.

Se identificaron cuatro compañías que registran la mayor cuota de mercado en el sector de curtido y adobo de pieles en Ecuador. Las empresas concentradas que se estudian en la presente investigación controlan el 73,12\% de la cuota de mercado, presentando un índice de Herfindahl-Hirschman de 2.010,68 para el año 2015.

En el período estudiado, las cuatro empresas con mayor concentración de mercado en el sector de curtido y adobo de cuero en promedio registraron un margen bruto de rentabilidad del 0,1391, es decir que, por cada dólar vendido existe una rentabilidad del 14\%. Estas cuatro empresas evidenciaron un incremento de sus niveles de rentabilidad en el mismo período a excepción del año 2014.

La Concentración de mercado expresada a través del Índice de HerfindahlHirschman registró un valor p estadísticamente significativo, siendo su valor de 0,0027 conjuntamente con la variable costos de producción. El modelo econométrico muestra que la parte de la rentabilidad no explicada por los costos de producción es explicada importantemente por los niveles de concentración de mercado, es decir que, este grupo de empresas obtienen beneficios por efecto de su capacidad para concentrar el mercado. Se aceptan las hipótesis de que la estructura de mercado imperfecta incide en los niveles de rentabilidad de las empresas líderes del sector de curtido y adobo de cuero y que los costos de producción inciden en los niveles de rentabilidad del oligopolio.

\section{Referencias}

Caballero, P. (2014). Gestión administrativa de la actividad comercial. Manual teórico. (Primera Ed.), Madrid: Editorial CEP.

Cardona Olaya, J., Martínez Carvajal, A., Velásquez Restrepo, S., \& López Fernández, Y. M. (2015). Análisis de los indicadores financieros del sector manufacturero del cuero y marroquería: Un estudio sobre las empresas colombianas. Informador Técnica.

Castaño Ríos, C. E., Acevedo Zuluaga, S., Madrid Ramírez, F., \& Soto Zuluaga, E. A. (2016). Rendimiento financiero en empresas productoras de cemento, cal y yeso de Antioquia en el período 2008 al 2013 y su relación con el PIB del sector manufacturero. Science of human, 8-36.

Duarte, J. (2014). Estrategias de entrada a un oligopolio: el caso de cementos andino. Universidad y empresa, 16(26), 115-136.

Instituto Nacional de Estadística y Censos (2012). Clasificación Nacional de Actividades Económicas. (https://goo.gl/CB7SvU).

Jaén, M. (2013). Economía industrial (Primera ed.). Almería, España: Universidad de Almería.

Jaén, M., Carretero, A., Amate, I., \& Piedra, L. (2013). Microeconomía básica (Primera ed.). Madrid, España: Septem Ediciones.

Lema, E. (2017). El oligopolio del sector curtiembre CIIU: C151101 y la rentabilidad de las empresas en el Ecuador (Tesis de grado). Ambato, Ecuador: Universidad Técnica de Ambato.

Navarro, J., Ocampo, C., \& Saumeth, L. (2013). Comportamiento oligopólico en el mercado mundial de aceite de palma 1961-2004. Ensayos de Economía, 26(48), 143-162.

Parkin, M., \& Loría, E. (2010). Microeconomía (Novena Ed.). México D.F.: Pearson Educación.

Servicio de Rentas Internas del Ecuador (2017). Estadísticas multidimensionales. (https://goo. gl/QuZzpr).

Superintendencia de Compañías del Ecuador. (2017). Portal de información. (https://goo.gl/i34FTQ).

Tanaka, G. (2005). Análisis de estados financieros para la toma de decisiones (Primera Ed.) Lima: Fondo Editorial de la Pontificia Universidad Católica del Perú. 